American Journal of Applied Sciences 8 (6): 644-651, 2011

ISSN 1546-9239

(C) 2011 Science Publications

\title{
Creating a Geographical Information Systems-based Spatial Profile for Exploring Health Services Supply and Demand
}

\author{
Abdulkader A. Murad \\ Department of Urban and Regional Planning, Faculty of Environmental Design, \\ King Abdulaziz University, Jeddah-21589, Saudi Arabia
}

\begin{abstract}
Problem statement: GIS has several techniques and functions that can be used for health services planning. Each one of these functions can be applied for different health related issues. Approach: The first part of this study reviews the relevant GIS functions that are used by health care planning researches and identifies the advantages of using these functions. The second part of the study focuses on presenting a GIS application created for the purpose of exploring the demand and supply on health services in Jeddah city, Saudi Arabia. A geodatabase is created for health services which covers the location and characteristics of health supply and demand. The created application covers three major analytical issues. The first issue is related to identifying the level of health services supply in Jeddah city. The location of health services is defined and classified based on the existing level of supply. The second issue of this application is related to defining the location and characteristics of health demand in Jeddah city. The locations of patients are defined and the actual catchment areas of health services are defined for this application. Accordingly, a demand based catchment area is created to define the growth and extent of health catchment area in Jeddah city. The third major analytical issue of this application is related to identifying the level of accessibility to health services in Jeddah city which is achieved using accessibility indicators scores. Results: The outputs of this application are useful for evaluating the location of health services supply and demand in Jeddah city. Conclusion: Using GIS in health care planning is recommended for health care planners in Jeddah city. The created application is considered as a spatial decision support system for health planners in Jeddah city.
\end{abstract}

Key words: Geographical Information Systems (GIS), health services, health demand, spatial analysis, spatial profile, GIS technology, overlay analysis, arc GIS software, point data, catchment area

\section{INTRODUCTION}

Health care facilities at any region can be divided into two main types that are known as primary health centers and hospitals. The former provides basic health care services and the latter provides services for specialist health treatment. Health authorities have always aimed to provide health care for all residents using a fair access policy that is characterized as providing the right service at the right time in the right place (Murad, 2006). To ensure adequate health care planning, health service planners and policy makers need accurate and reliable measures of health facilities so that true services shortage areas can be accurately identified and resources allocated to those needy areas to alleviate the problem. Health care planning in a given location is influenced by many factors, including the availability of health services in the area (supply), the number of people living in that location (demand), the population's health status, the socio-economic and financial resources available to the population, people's knowledge about health and the health care system and geographical impedance between population and health services (Luo and Qi, 2009). Among the many factors that influence health care services, two of them are critical: physician supply and population demand. Both of these are spatially distributed, but it is rare that their distributions perfectly match (Luo, 2004).

One of the main issues that health planners need to cover at any built up area is related to evaluating health accessibility. Measures of geographical accessibility have also been proposed and critiqued in the planning and medical geography literature (Guagliardo et al., 2004). For example, Khamis and Hanoon (2010) examine the relationship between children mortality, prosperity and education to create a clear picture of health status of three indicators of children mortality: stillbirth, neonatal and infant. Such measures range from the conceptually simple counting of the number of facilities within a specified distance from a given 
location to more sophisticated spatial interaction models. These measures can be implemented using Geographical Information Systems (GIS). GIS plays an essential role in helping public health organizations understand population health and make decisions. GIS has been used to create layers that can show decision makers in a simple, easy and flexible manner (Fadda et al., 2008). With the powerful tools and solutions that GIS technology brings to the desktop, health planners can improve understanding of community health needs and design effective interventions. GIS technology offers varied solutions including ones that improve field data collection and reporting and others that support disease surveillance and analysis with online mapping and spatial statistics. In addition, GIS, improve the ability to communicate with several health situation, such as environmental contamination, to decision makers.

Geographical Information Systems (GIS) can be used for several studies. For example, GIS plays a key and important role in preparing Prayer time map (Aghighi et al., 2008) and it is used for simulation planning for sustainable use of land resources (Yacouba et al., 2009) and for analyzing the concept of spatialfunction in courtyard house layout and its influence to the cultural and social activities (Mustafa and Hassan, 2010). In addition, Liaghat and Balasundram (2010) indicated that Precision Agriculture (PA) can now based on innovative systems approach and these new systems approach depends on a combination of fundamental technologies such as Geographic Information System (GIS), Global Positioning System (GPS), computer modeling, ground based/airborne/satellite remote sensing, variable rate technology and advanced information processing for timely in-season and between season crop management. Examples of GIS studies in health care planning include examining disease rates, examining variations in health and the use of health services. Wilkinson et al. (1998) addresses the potential applications of GIS in health geographical studies. These applications are: disease mapping and geographical correlation studies, patterns of health service use and access, environmental hazards and disease clusters and the modeling of the health impacts of environmental hazards. Jacquez (1998), added that GIS could be used for exposure assessment, identification of study populations, disease mapping and public health surveillance. There are several examples in the literature that discuss the potential GIS application in health care facility planning. For example, GIS is used for monitoring vector borne disease, water borne diseases, environmental health, modeling exposure to electromagnetic fields, quantifying lead hazards in a neighborhood, predicting child pedestrian injuries and for the analysis of disease policy and planning (Coggon et al., 2003).

Rytkonen et al. (2003), discussed an interesting GIS application for analysing the incidence of type 1 diabetes among children in Finland. They observed the incidence of type 1 diabetes per 100,000 persons separately in urban areas, urban-adjacent rural areas, rural health and remote areas. Cerrito presented another GIS based health study, investigating the relationship between environmental factors and the need for the treatment of lung problems. It is considered an interesting case, demonstrating how the data mining of GIS, combined with healthcare outcomes, can be effective in modifying clinical research. One example of using GIS and GPS in health care is found in Guagliardo et al. (2004), where these technologies are used to map out residence activity spaces, using symbols and standard deviational ellipses and sites where diabetes information has the potential to be welcomed, for a sample of low income females and males. This example shows how 'prevention of diabetes' projects can use GPS and GIS tools to collect and record the activity spaces of 121 participants and demonstrates how this approach can be used by healthcare providers and researchers to implement a community-based diabetes prevention programme.

Krause et al. (2005), analyze the availability of dentists in Mississippi by county over four decades to determine the geographic distribution of dentists, shifts in their distribution over time and how this distribution relates to population demographics using standardized $\mathrm{z}$ scores and GIS technology. Their results can assist current and future practicing dentists, dental school administrators and policy-makers in making informed decisions for determining suitable practice locations, dental school admissions criteria and areas to target for public health initiatives.

A combination of multi-attribute, temporal and comparative visualization techniques is used to highlight the strengths and identify the weaknesses of a partition (Shanbhag et al., 2005). GIS data visualization (Kaneko et al., 2003) and statistical techniques suggest that certain policy definitions of underserved areas may not be effective in defining areas of nursing shortages (Courtney, 2005). Klauss et al. (2005), create and characterize population-based hospital service areas for Switzerland and visualize results as maps in a GIS. Hospital service areas and health utilization indices provide valuable information for health care planning. Vinnakota and Lam (2006), use GIS to integrate data about the socioeconomic inequality of mortality of colorectal, lung, breast and prostate cancers in the USA defined at different spatial resolutions as well as to 
visualize and analyze the results from the association of rule mining process with GIS technology in order to identify regions that need further attention.

The purpose of this study is to present a GIS-based spatial profile created for exploring health care services supply and demand in Jeddah city, Saudi Arabia. The profile covers three important health care planning issues. These are covering health supply classification, health demand classification and health care accessibility.

GIS tools for health care applications: The GIS software packages such as ArcGISArcInfo and ArcGISArcview (ESRI, Redlands, Ca, USA) include many functions and tools designed for health care studies. These functions can be used for the visualization of health data, spatial analysis and modeling. They are crucially important in any health-oriented study as they enable better decision-making by providing health status and needs for an area or region from a spatial point of view. GIS based analysis and modeling functions can be classified according to data model types (vector or raster data). The density function, for instance, can be used as point data for the location of health care centers, for the estimation of spatial variations in disease risk and for a spatial demonstration of point-pattern intensity. The output of the density function is more useful for pattern recognition than for the location of individual features and particularly, for mapping areas of different sizes such as census tracts or counties. Two main methods are used for calculating density in the ArcGIS software. The first one simply counts the features within a search radius, while the second one is a weighted method used as a mathematical function (e.g., the Kernel function) to emphasize features closer to the centre of raster cells. Health care data such as location of patients can be analyzed by this function to identify the spatial variation of these patients in any area. In addition to apply GIS for defining spatial classification of health supply, it can also be used to for multiple data classification or so-called multiple queries. This type of GIS function reveals features satisfying a criterion with more than one parameter, e.g. to identify health centers with many physicians but no dentist, or with inadequate number of general staff. Multiple queries can be made in GIS using logical operations dealing directly with the database allowing the user to identify and select features by a special set of criteria. In most GIS applications, features are identified and selected from the database and highlighted on the map. Such selected features can be saved in a new coverage for further analysis. Health studies can use several GIS based neighborhood statistics functions such as the focal mean, the focal sum or the focal range, which computes the required statistics for either rectangular or circular neighborhood shapes. The kernel function is another important GIS function which can be used for exploring health data. This function is useful for calculating the spread out of health incidence over a surface. The measured quantity of health data sent (line or point) is distributed throughout a landscape and a density value is calculated for each cell in the raster output. Health researchers can use the above GIS data exploratory functions to find answers to questions such as whether there is a cluster of health events at any location.

ArcGIS software has a very useful extension known as the Geostatistical Analyst, which can be used for modeling any point based data, such as the location of patients and can easily create a continuous surface from measured sample points stored in a point-feature layer. It derives a surface using the values from the measured locations to predict values for each location in the landscape (Johnston, 2001). Kriging is one of the useful GIS functions that can be used for modeling health point data. It is considered as one of the deterministic interpolation methods which capable of producing a prediction surface and providing some measures of the certainty or accuracy of a prediction.

Gholizadeh et al. (2009) added that environmental data were interpolated geostatistically by kriging technique using ArcGIS 9.2 through spatial analysis extension on semivariogram results for Geostatistical Software(GS+). Health studies that look into the relationship between air pollution and health status can use this function to define air quality, based on sample measured points.

Geo-coding function: One of the important issues related to health care planning is to identify the location of health care needs. Usually health authorities have a list of their registered patients and therefore, they need to plot these lists on their geographical area. GIS can be used in this manner because it has the tool of converting any address data into point coverage through the geo-coding function. This function is used by several applications to create points on a map from a table of addresses. For example, Fadda et al. (2008) indicate that GIS can be used for Building a relational database system that includes GIS layers and RS image maps and managing them, for producing digital image maps for Jordan (Al-Salt) and for evaluating the validity and the appropriateness of data fusion in relation to visualization.

To geo-code addresses, users should have reference coverage to serve as a base map (the reference coverage is usually a street theme with attributes that specify the street name, street type and range of addresses that 
occur a long each street). Once the reference coverage is prepared, any address table, which contains patient addresses, can be covered into points on the map. Many GIS softwares have a specific module for handling the address data. For example ArcGIS of ESRI uses address information in the attribute table of the reference data to figure out where to locate address points. This is achieved by using the geo-coding service that is a file that specifies the reference data and its relevant attributes, the relevant attributes, the relevant attributes from the address table and various geo-coding rules and tolerances (Ormsby et al., 2010).

Overlay analysis: The concept of overlay analysis is one of the major GIS procedures that are used by several studies. It manipulates spatial data organized in different layers to create combined spatial features according to logical conditions specified in Boolean algebra. GIS stores data is layers that can be retrieved and overlaid one on another to answer questions such as which sites are most suitable for development of variety types. Such site is determined by checking the suitability of location on each of a number of key criteria. The locations that pass the test on all these criteria are the suitable for development (Birkin, 1996). Many GIS softwares have several overlay analysis functions, which applied on raster or nectar data models. In the case of feature-based data, Union, Interact and Identity are the major polygon overlay functions. These are used by planners to get new coverage that satisfy the required criteria. The overlay procedure is often undertaken in conjunction with spatial buffering, another standard feature of a proprietary GIS (Birkin, 1996). It enables the user to determine an area at a chosen distance shown either a point location (e.g., health center) or a line feature (e.g., a road or a lines) (ibid). The output buffer function is a circle for points, a corridor for lines and a polygon for polygons.

Network analysis: The third main GIS tool that is considered to be very useful in health care studies is the Network Analysis. It is one of the most frequently used components of a GIS in the utilities and transportation planning fields. It can find answers to questions such as what is the shortest path between location $\mathrm{X}$ and $\mathrm{Y}$. Such analysis is known as routing or path finding which determine the minimum cost path through a network between a given origin and destination. This function can be used in the health studies for defining the shortest path between patient location and health center. The resulted path can be presented to the ambulance driver together with the direction file that describes step by step the best routes for getting fast to such patient.
Allocation is another important function available in GIS Network analysis that facilitates the modeling of resource distribution through a spatial network and the determination of service zone. For example, the number of pupils in a district can be assigned to the nearest network link and then in turn allocated to school based on user defined rules concerning maximum travel distance (Birkin, 1996). The use of Allocate function can be used in health studies in creating health center catchment area.

\section{MATERIALS AND METHODS}

One of the main tasks that should be looked at carefully during the building of any GIS application, is regarding creating the required data and then integrating these data within the GIS application. These data fall into three main GIS data features known as points, lines and polygons. Point data are restored as a single $\mathrm{x}, \mathrm{y}$ coordinate, with attributes describing the conditions of these points. Usually geographical features that are too small to be depicted as lines or areas, are created in GIS as points data. For this application, the location of health centers in Jeddah city is created as a point feature and all attribute data about health centers are saved in the attribute table of this file. The second main GIS data feature is the line feature, which has a one dimensional shape that represents geographical features too narrow to depict as area (Zeiler, 1999). GIS software stores lines as a series of ordered $\mathrm{x}, \mathrm{y}$ coordinates, with the relevant attributes. For the presented application, the road network of Jeddah city is represented as a line feature, with attributes regarding the length and type of each road in this city.

\section{RESULTS AND DISCUSSION}

Health services at Jeddah city: The first issue is related to building a GIS model that describes the existing situation of some of health services supply (physicians and dentists) in Jeddah city. This model is important for health planners because it helps them in having a visual view about their facilities and services. It can be used either to identify problem areas, or to select city parts that need some detailed health studies. Several types of data were used to construct this model. The data were about existing location of health services in Jeddah city. All health centers are plotted on GIS as point features map and classified based on their supply or service. There are 11 types of health services supply for each centre. They are the following: 1 - physicians (general practitioners, GPs), 2 - family physicians, 3 - dentists, 4 nurses, 5- midwives, 6-pharmacists (pharmacologists), 7 - 
laboratory technicians, 8-x-ray technicians, 9administrators, 10 - servants and 11 - others.

Each health centre presents with various amounts of the abovementioned supply or types of health services. In this study, we have selected physicians and dentists as examples of health services supply provided in health centers. The same visualizing techniques can be implemented with the rest types of health services supply, e.g. pharmacologists and laboratory technicians. Identifying the relationship between supply and demand/utilization is very important for health care planners. GIS helps in describing and classifying any types of features, e.g. points, lines or polygons based on values of attributes' data. This technique is applied on health centre shape file and each type of health services supply data is classified according to its values.

Figure 1 shows a classification model for a health centre based on the number of physicians while Fig. 2 demonstrates a classification of health centers based on the number of dentists. Each of these figures provides a visual evaluation and comparison between health centers based on their existing supply. Visualization of health information is, usually, the first step in health research. It can be very useful in obtaining the first overview of data distributions and possible spatial patterns. Another important issue dealing with health data visualization is related to the concerns of the variable size of spatial units. It means that large areas with low population rates can attract the analyst even more than smaller areas receiving larger population figures. To overcome this visual problem, GIS can be used for visualizing maps that show features as proportions to their relative health data. Accordingly, features that have large population values are presented in GIS with larger symbols; meanwhile, low population features are shown with smaller icons or symbols.

Looking at the number of physicians at a given health centre, it can be stated that each health center has different amount of physicians or dentists. Some centers such as Alsafa Center and Gulail Center engage a larger amount of physicians while in other ones such as Alsharafia Center and Althayuar Center this number is smaller. The situation is similar when dentists are concerned. In some centers there are two dentists, e.g. in Alazizia Center while in other ones, e.g. in Alselamainah Center and Almarwah Center there are no dentists at all. There are various reasons for this uneven health supply in Jeddah city. One of them is related to the size of existing demand.

Along with defining spatial classification of health supply, GIS is used to classify more types of this supply. This is known as multiple data classification, the so-called multiple queries. This type of GIS functions can reveal features satisfying a criterion with more than one parameter.

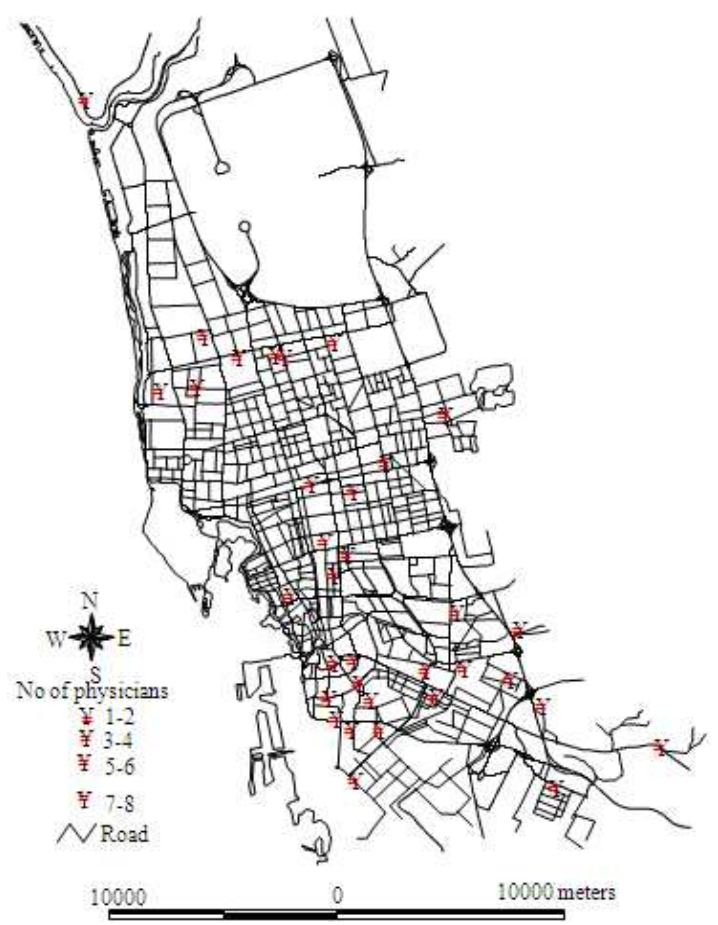

Fig. 1: Classification of health centers based on number of physicians

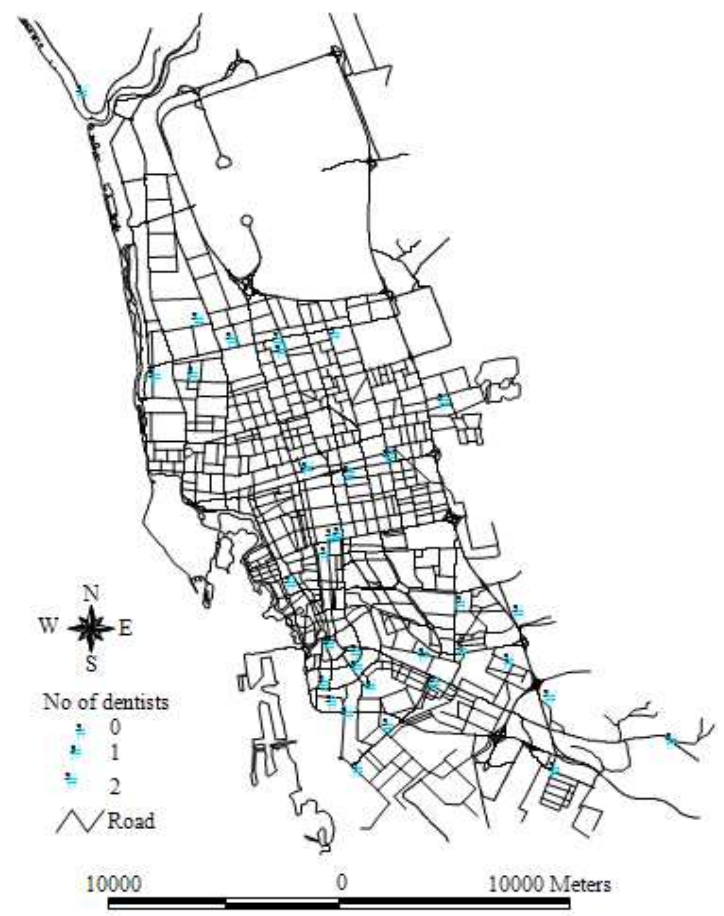

Fig. 2: Classification of health centers based on number of dentists 
An example is to find out health centers with a large number of physicians but without any dentists or with less servants. Multiple queries can be made in GIS using logical operations dealing directly with the database and allowing the user to identify and select features by a special set of criteria In most GIS applications, features are identified and selected from the database and highlighted on the map according to a combination of several conditions. The selected features can be saved in a new coverage for further analysis.

At presence, two different multiple queries have been used to search in the health centers. First, these of them have been detected which are owned by the health authority and engage more than 3 physicians. This search produces an output showing health centre providing large amount of services but not built on a public building. This output can be used by the health authority to decide about the health centers that should have the priority in having a new public building. Figure 3 describes the results of this multiple query search and indicates that several health centers in Jeddah city are located on rented buildings and engage a lot of physicians. They are mainly located it the southern area of the city (e.g., Ghulail and Aljameaa centers). However, there are other centers located in the northern part of Jeddah city (e.g., Alzahraa and Alsalamah centers) with the same characteristics.

The second multiple search model is created to define the health centers providing health care service by family physicians and dentists. It defines the health centers delivering large and various types of health services to the public (Fig. 4). Obviously, some of them such as Alrabwa, Alzahra and Alnaeem centers meet these requirements. However, some other centers (e.g., Alsafa and Alsohaifa) do not engage dentists and family physicians at all. The local health authority can therefore use this technique to find quick and clear answers about any issues related to quality and quantity of health services delivered at Jeddah city.

Health demand at Jeddah city: The aim of this model is define the spatial distribution of health demand in Jeddah city. In order to cover this issue, a sample of 100 patients is made for the presented application. A survey is made to define the location of health demand and to identify the profile of patients in Jeddah city. The profile covers location of patients, reason for visiting clinic, health status and income of patients. The collected data were entered into the GIS as point data feature and then classified based on the relevant attributes that are available in the database.

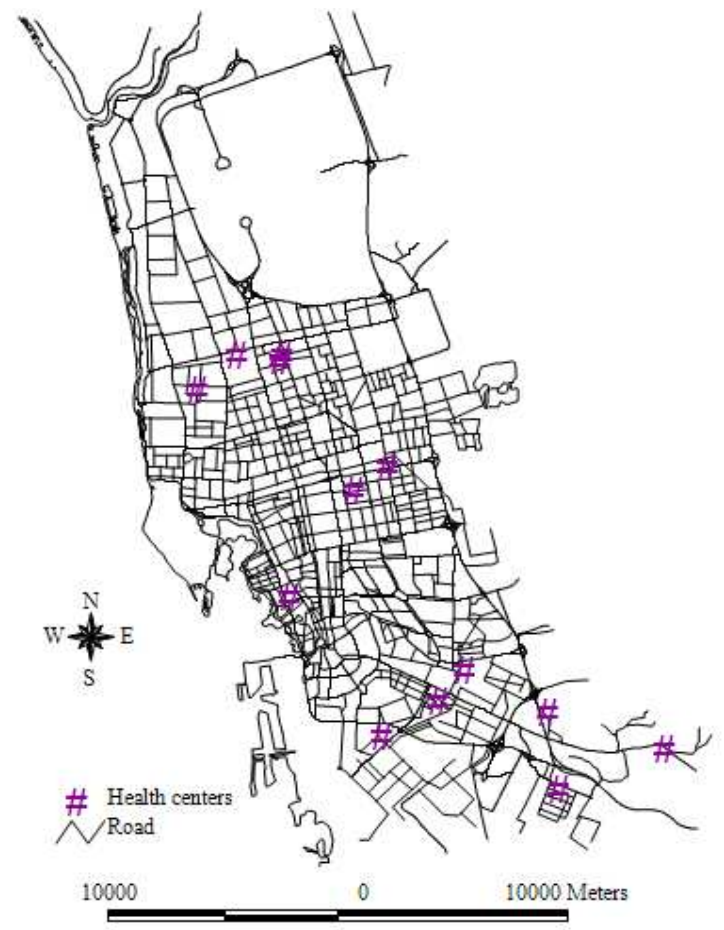

Fig. 3: Rented health centers with more than 3 physicians

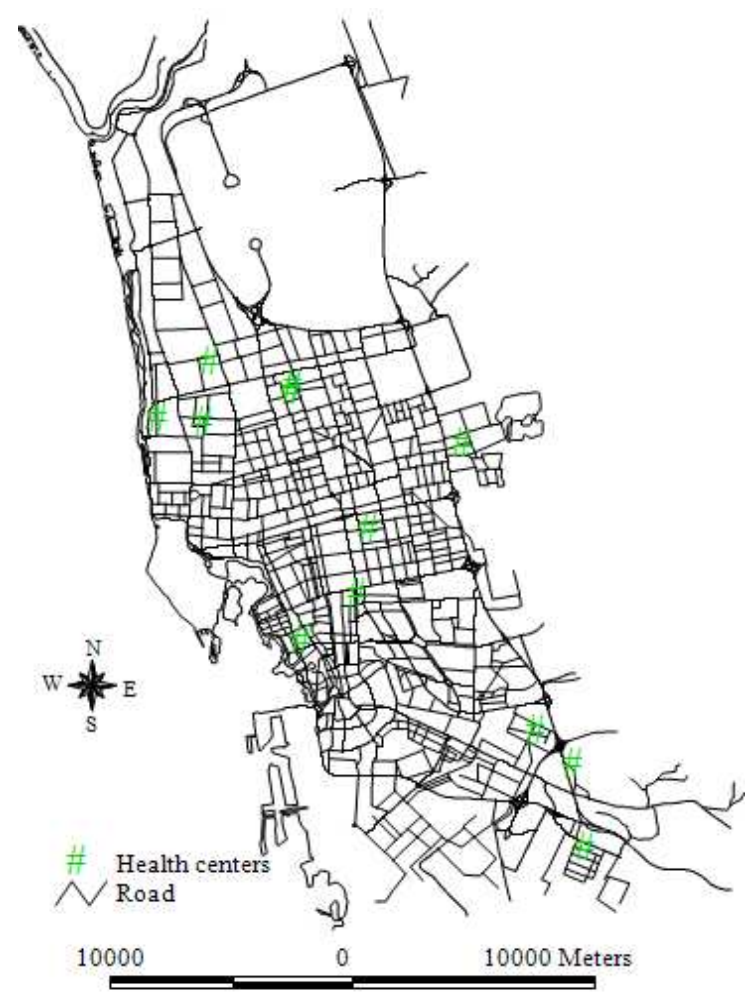

Fig. 4: Health centers with family doctors and dentists 
Am. J. Applied Sci., 8 (6): 644-651, 2011

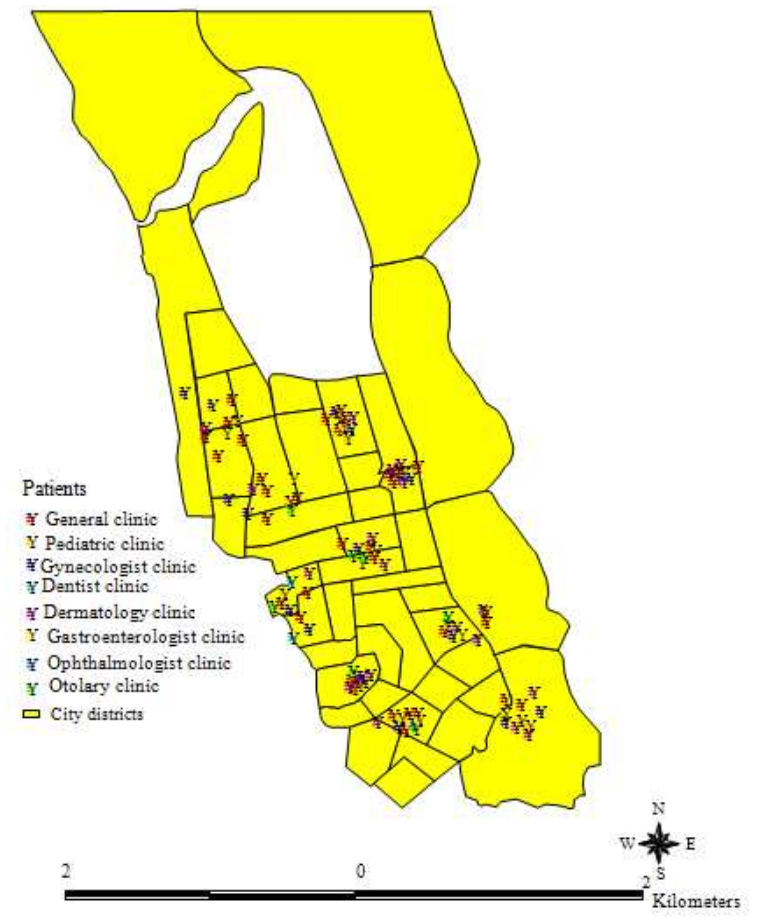

Fig. 5: Classification of health demand based on clinics types

The results of this profile indicate that $40 \%$ of patients are visiting general doctor clinics, $20 \%$ are visiting pediatric clinic and $20 \%$ are visiting dentist clinic and $20 \%$ are visiting other clinics such as NET clinic, gyna clinic. Figure 5 defines the spatial classification of patents at Jeddah city and classifies patients based on clinics types. Health planners can use this model to get a clear understanding about the typology of health demand in Jeddah city and to allocate health supply according to the results of this output.

Accessibility to health centers: The literature on accessibility measures showed a need for quantitative indicators of accessibility for different kinds of public services including health care. Such indicators would serve as instruments in the comparisons of accessibility in different parts of the region and in the evaluation of alternative plans for new service facilities and transportation links. Examples of accessibility indicators are: provider-to-population ratio, distance to the nearest provider, average distance to a set of providers and gravitational models of provider influence (Guagliardo et al., 2004). Each one of these indicators can be used to evaluate accessibility of health centers. The presented application has selected distance to provider method and produces accessibility indicators to health centers in Jeddah city.

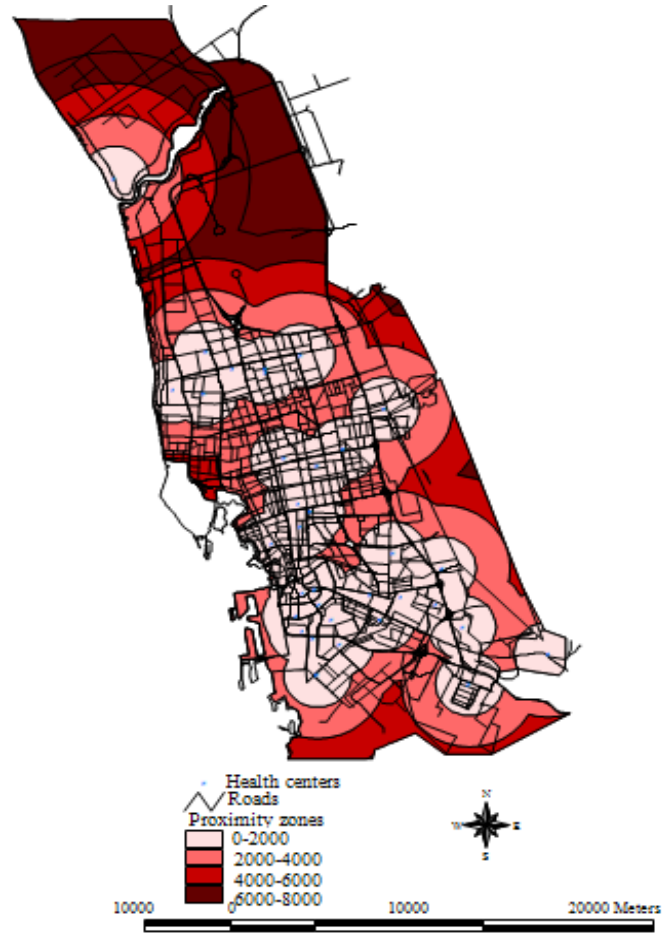

Fig. 6: Proximity to health centers at Jeddah city

One way of defining accessibility to health centers is by knowing how far patients live from their nearest centers. Based on local standards, every health center should cover a catchment area extending $2 \mathrm{KM}$ radius wide. In order to define the level of accessibly to health centers, GIS proximity analysis was used and the output of this model (Fig. 6) classifies the city into deferent zones based on the distance between clinic location and city districts. Based on this output, several parts of the city are located at areas with more than $2 \mathrm{KM}$ accessibility zone. These areas are mainly situated north and east of the city with some to the west.

\section{CONCLUSION}

This study discusses a GIS application for health centers in Jeddah city. The application covers three main health care planning issues. These are called identifying the level of health services supply, defining the location and characteristics of health demand and identifying the level of accessibility to health services in Jeddah city. Each one of these issues has a direct spatial dimension. Therefore, the use of GIS for analyzing and manipulating health data was of greater value and benefit. Different data sets were collected and captured using ArcGIS software. The outputs of this application provide health planners of Jeddah city with spatial tools for evaluating the existing location of health supply and demand. 


\section{REFERENCES}

Aghighi, H., A. Alimohammadi and M.S. Ghahareh, 2008. Prayer times modeling with GIS: A case study for Iran and its surrounding. J. Comput. Sci., 4: 807-814. DOI: 10.3844 jcssp.2008.807.814

Birkin, M., 1996. Intelligent GIS: Location decisions and strategic planning. 1st Edn., GeoInformtion International, USA., ISBN-10: 0470236140, pp: 292.

Coggon, D., R. Geoffrey and D.J.P. Barker, 2003. Epidemiology for the Uninitiated. 5th Edn., John Wiley and Sons, USA., ISBN-10: 0727916041, pp: 73.

Courtney, K.L., 2005. Visualizing nursing workforce distribution: Policy evaluation using geographic information systems. Int. J. Med. Inform., 74: 980988. PMID: 16085452

Fadda, E.H.R., M. Kakish and E.J. Akawwib, 2008. Relational GIS and remote sensing database system for al-salt area, Jordan. Am. J. Eng. Applied Sci., 1: 241-247. DOI: 10.3844/ajeassp.2008.241.247

Gholizadeh, A, M.S.M. Amin, A.R. Anuar and W. Aimrun, 2009. Evaluation of leaf total nitrogen content for nitrogen management in a malaysian paddy field by using soil plant analysis development chlorophyll meter. Am. J. Agric. Biol. Sci., 4: 278-282. DOI: 10.3844/ajabssp.2009.278.282

Guagliardo, M.F., C.R. Ronico, I. Cheung, E. Chacko and J.G. Josef, 2004. Physician accessibility: An urban case study of pediatric providers. Health Place, 10: 273-283. PMID: 15177201

Jacquez, G., 1998. GIS as an Enabling Technology. In: GIS and Health, Gathell, A. and M. Loytonen, (Eds.). Houghton Mifflin Harcourt, ISBN-10: 0748407790, pp: 17-28.

Johnston, K., 2001. Using ArcGIS Geostatistical Analyst. Environmental Systems Research Institute, USA., ISBN-10: 1589480066, pp: 300.

Kaneko, Y., T. Takano and K. Nakamura, 2003. Visual localisation of community health needs to rational decision-making in public health services. Health Place, 9: 241-251. PMID: 12810331

Khamis, F.G. and M.F. Hanoon, 2010. On studying the linkage between children mortality, prosperity and education using fully latent models. J. Soc. Sci., 6: 33-40. DOI: 10.3844/jssp.2010.33.40

Klauss, G., L. Staub, M. Widmer and A. Busato, 2005. Hospital service areas-a new tool for health care planning in Switzerland. BMC Health Serv. Res., 5: 33-33. PMID: 15882463

Krause, D., D.A. Frate and W.L. May, 2005, Demographics and distribution of dentists in Mississippi: A dental work force study. J. Am. Dent. Assoc., 136: 668-677. PMID: 15966657
Liaghat, S. and S.K. Balasundram, 2010. A review: The role of remote sensing in precision agriculture. Am. J. Agric. Biol. Sci., 5: 50-55. DOI: 10.3844/ajabssp.2010.50.55

Luo, W. and Y. Qi, 2009. An enhanced two-step floating catchment area (E2SFCA) method for measuring spatial accessibility to primary care physicians. Health Place, 15: 1100-1107. DOI: 10.1016/j.healthplace.2009.06.002

Murad, A.A, 2008. Creating a GIS-based epidemiological application for jeddah city. Int. J. Health Care Technol. Manage., 9: 540-551. DOI: 10.1504/IJHTM.2008.020204

Murad, A.A., 2006. Creating a GIS application for health services at Jeddah city. Comput. Biol. Med., 37: 879-889. DOI: 10.1016/j.compbiomed.2006.09.006

Mustafa, F.A. and A.S. Hassan, 2010. Spatialfunctional analysis of kurdish courtyard houses in Erbil city. Am. J. Eng. Applied Sci., 3: 560-568. DOI: 10.3844 /ajeassp.2010.560.568

Ormsby, T., E.J. Napoleon, R. Burke, C. Groessl and L. Bowden, 2010. Getting to know Arc GIS desktop. 2nd Edn., ESRI Press, Canada, ISBN-10: 1589482603, pp: 592.

Rytkonen, M., E. Moltchanova, J. Ranta, O. Taskinen and J. Tuomilehto et al., 2003. The incidence of type 1 diabetes among children in Finland--ruralurban difference. Health Place, 9: 315-325. PMID: 14499216

Shanbhag, P., P. Rheingans and M. Desjardins, 2005, Temporal Visualization of Planning Polygons for Efficient Partitioning of Geo-Spatial Data. Proceedings of the IEEE Symposium on Information Visualization, Oct. 23-25, Maryland University, USA., pp: 211-218. DOI: 10.1109/INFVIS.2005.1532149

Vinnakota, S. and N.S. Lam, 2006. Socioeconomic inequality of cancer mortality in the United States: A spatial data mining approach. Int. J. Health Geogr. DOI: 10.1186/1476-072X-5-9

Wilkinson, P., C. Grundy, M. Landon and S. Stevenson, 1998. GIS in Public Health. In: GIS and Health. Gatrell, A. and M. Loytonen, (Eds.). Taylor and Francis, London, UK., pp: 179- 189.

Yacouba, D., H. Guangdao and A.H. Abdulahi, 2009. Simulation planning for sustainable use of land resources: Case study in Diamou. J. Math. Stat., 5: 15-23. DOI: 10.3844/jmssp.2009.15.23

Zeiler, M., 1999. Modeling our World: The ESRI Guide to Geodatabase Design. 1st Edn., ESRI, USA., ISBN-10: 1879102625, pp: 199. 